\title{
Intravenous lidocaine for the treatment of acute pain in the emergency department
}

\section{Brendan Michael Fitzpatrick, Michael Eugene Mullins \\ Division of Emergency Medicine, Washington University School of Medicine in St. Louis, St. Louis, MO, USA}

Objective To evaluate intravenous lidocaine's safety and efficacy as an analgesic agent in the treatment of a variety of painful conditions presenting to the emergency department.

Methods This case series identified seventeen patients who received lidocaine over a six month period and recorded demographic data, amount of lidocaine administered, the amount of opioid medication administered before and after lidocaine, pre- and post-lidocaine pain scores, and any qualitative descriptors of the patient's pain recorded in the record. Side effects and adverse events were also recorded.

Results Of the seven patients who had a pre- and post-lidocaine pain score recorded, the mean reduction was 3 points on a 10 point scale. Patients who received lidocaine used less opioid medication. One patient received an improperly high dose of lidocaine and suffered a brief seizure and cardiac arrest, but was quickly resuscitated.

Conclusion This series suggests that lidocaine may be a useful adjunct in the treatment of acutely painful conditions in the emergency department.

Keywords Lidocaine; Analgesia; Pain control
elSSN: 2383-4625

Received: 2 January 2016

Revised: 5 February 2016

Accepted: 6 February 2016

Correspondence to:

Brendan Michael Fitzpatrick

Division of Emergency Medicine, Washington University School of Medicine in St. Louis, 660 E. Euclid Ave, CB 8072, St. Louis, MO 63110, USA

E-mail: fitzpatrickb@wusm.wustl.edu

\footnotetext{
Capsule Summary

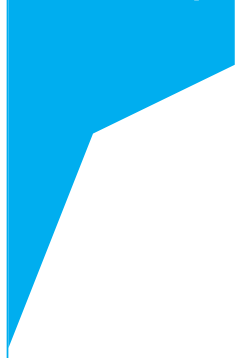

\section{What is already known}

Intravenous lidocaine has been used to treat pain in postoperative patients, opioid-refractory chronic pain, and oncologic pain. Recent data suggests intravenous lidocaine may also be beneficial in treating acutely painful conditions in the emergency department.

What is new in the current study

This case series reviewed intravenous lidocaine's effectiveness and safety in treating a variety of painful conditions in the emergency department, including but not limited to acute fracture pain, abdominal pain, sickle cell pain crisis, burns, contusions, and penetrating trauma. Lidocaine seemed to reduce pain and decreased the amount of opioids required after its administration. When used in appropriate doses, there were no significant side effects. This case series adds to the body of literature suggesting that intravenous lidocaine might be considered as an adjunct to acute pain management in the emergency department across a spectrum of painful conditions.
}

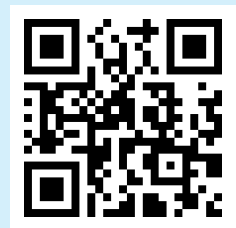

How to cite this article:

Fitzpatrick BM, Mullins ME. Intravenous lidocaine for the treatment of acute pain in the emergency department. Clin Exp Emerg Med 2016;3(2):105-108.

This is an Open Access article distributed under the terms of the Creative Commons Attribution Non-Commercial License (http:// creativecommons.org/licenses/by-nc/4.0/). 


\section{INTRODUCTION}

Emergency physicians are familiar with lidocaine's use as a local anesthetic. For many years, lidocaine was also part of the advanced cardiac life support algorithm as an anti-arrhythmic, ${ }^{1}$ and is even still recommended in some airway texts as a neuroprotective agent used prior to airway management in patients with elevated intracranial pressure. ${ }^{2}$

However, lidocaine's use as an intravenous analgesic has been far less popular in emergency medicine than in other areas of medicine. Intravenous lidocaine has been used for decades in treating oncologic pain, ${ }^{3}$ post-surgical pain management, ${ }_{1}^{4}$ and in chronic opioid-refractory pain. ${ }^{5}$ More recently, trials have shown analgesic efficacy in the treatment of painful conditions in the emergency department; particularly in the treatment of renal colic ${ }^{6,7}$ and limb ischemia. ${ }^{8}$

The objectives of this case series were to evaluate intravenous lidocaine's safety and efficacy as an analgesic agent in the treatment of a variety of acutely painful conditions presenting to a tertiary urban academic emergency department.

\section{METHODS}

Patients who received intravenous lidocaine for acute pain during a six month period from 1 January 2012 through 1 July 2012 were eligible for inclusion in this series. We excluded patients who received intravenous lidocaine for other reasons, e.g. arrhythmia, neuroprotection for intubation. We identified eligible patients by reviewing the institution's pharmacy data during this time. The study was approved by the institutional review board.

Data recorded included the patient's age, sex, chief complaint and/or diagnosis, disposition, total amount of intravenous lidocaine received in milligrams, triage pain score, post-lidocaine pain score, and any qualitative descriptors used by the patients to describe their pain before and after lidocaine. Additionally, the amount of opioids administered before and after initial lidocaine in morphine equivalents, and any recorded side effects or adverse events from lidocaine were also documented.

\section{RESULTS}

Seventeen patients met the inclusion criteria. Seven women and ten men comprised the study group, with a median age of 48 years and an age range of 23 to 81 years. The most common diagnosis and cause of pain was an acute fracture in 5 patients, followed by sickle cell pain crisis, acute back pain, and abdominal pain, with 2 patients each. Other diagnoses were trigeminal neuralgia, acute ischemic foot, herpes gingostomatitis, foot contusion after motor vehicle collision, grease burn, and a gunshot wound to the hand. Ten of the patients were admitted, six were discharged home, and the patient with the ischemic foot underwent emergency surgery.

The mean dose of total lidocaine was $148.53 \mathrm{mg}$, with a median and mode of 100, and range of 75 to $400 \mathrm{mg}$. Six of the seventeen patients received two doses of lidocaine, and the other eleven received only one dose. Only seven patients had a pain score recorded before and after the administration of lidocaine. Two of these seven rated their pain as a 9/10, and five rated their pain as a 10/10 prior to the administration of lidocaine. The average pain reduction was 3 in these seven patients. One patient reported no change in their 10/10 pain, and another noted an improvement from 10 to 5 .

Reviewing nursing and provider descriptors of pain, several had patient quotations that indicated some improvement in their pain. Two patients were quoted as saying their pain was "better", another the pain was "easing up", and one patient was quoted as saying "I feel fine". Providers used the terms "improvement", "much better", and "better". Two patients described their pain as aching both before and after lidocaine, but there was nothing recorded about the pain's intensity. Three patients were documented as sleeping after receiving lidocaine.

The patients who received intravenous lidocaine received a total of $19.07 \mathrm{mg}$ of morphine-equivalent opioids. The mean total amount of opioid received prior to lidocaine was 11.1 and $8.5 \mathrm{mg}$ after lidocaine.

One patient suffered a serious adverse effect from intravenous lidocaine. The treating physician mistakenly free-text ordered lidocaine $100 \mathrm{mg}$ and mistakenly approved when the nurse presented $100 \mathrm{~mL}$ of $1 \%$ lidocaine, which was 5 vials of $20 \mathrm{~mL} 1 \%$ lidocaine. At the end of the second vial, which was a cumulative dose of $400 \mathrm{mg}$ of lidocaine, the patient seized. He soon became bradycardic, and eventually had a brief cardiac arrest. He was resuscitated successfully and made a full neurologic recovery. The dose of $400 \mathrm{mg}$ is four times greater than the next largest single dose given, $100 \mathrm{mg}$, and exceeds the usual studied dose. Root cause analysis determined that the physician was unfamiliar with the correct dosing of lidocaine.

\section{DISCUSSION}

Intravenous lidocaine has shown promise in treating acutely painful conditions, and in others it has shown no benefit. The purpose of this case series was to generate hypotheses for future areas of investigation. During a six-month-period, patients with fractures, sickle cell vasoocclusive crises, abdominal pain, neuropathic pain, 
and even mucocutaneous ulcers were treated with intravenous lidocaine. The results suggest that some patients experienced less pain after administration of this agent.

Lidocaine is an aminoethylamide that acts primarily as a sodium channel inhibitor, and exerts its effects as a local anesthetic by blocking sodium influx and halting action potential conduction. It blocks both open and inactivated sodium channels, with a greater effect seen at the already depolarized channels secondary to a greater positive resting potential. Thus, nerves that are repetitively stimulated such as those that are ischemic are more affected by lidocaine than non-stimulated nerves. ${ }^{9}$ Locally, this block occurs at the $A \beta, A \delta$, or $C$ primary afferent nerves, and at high enough concentration, causes a complete neural blockade in both injured and uninjured nerves. Systemic lidocaine, however, does not completely block conduction of these fibers if they are healthy. Instead, its effects occur primarily at acutely injured nerves that have a tonic action potential discharge..$^{10} \mathrm{~A}$ similar effect occurs in animal models at damaged dorsal root basal ganglia, in which systemic lidocaine reduced sympathetic noradrenergic sprouting. This benefit lasted for 7 days after the cessation of lidocaine. ${ }^{11}$

In the emergency setting, Soleimanpour et al. ${ }^{6}$ conducted an open label case series of patients treated with lidocaine $1 \mathrm{mg} / \mathrm{kg}$ for relief of renal colic. Based upon favorable results, they conducted a randomized trial comparing lidocaine $1 \mathrm{mg} / \mathrm{kg}$ to morphine $0.1 \mathrm{mg} / \mathrm{kg}$ for relief of renal colic and found that lidocaine achieve better pain reduction in the first two hours after drug administration. Intravenous lidocaine has shown efficacy in treating limb ischemia, ${ }_{1}^{8}$ neuropathic pain, postoperative abdominal pain, and headache. ${ }^{12}$ However, it had no benefit in treating burns and radicular back pain. ${ }^{13,14}$ It would seem, then, that the role of intravenous lidocaine in the treatment of acute pain is unclear.

This case series reviewed the use of intravenous lidocaine for a variety of painful complaints, with varying success. Some patients reported significant decreased levels of pain and reduced administration of opioids. Lidocaine, when administered at doses up to $3 \mathrm{mg} / \mathrm{kg}$, was well-tolerated by patients. The single significant adverse event occurred at a dose of $3.66 \mathrm{mg} / \mathrm{kg}$, and like any medication, care must be taken to educate physicians and nurses regarding the appropriate dosing.

It may be that lidocaine will be found to be a useful adjunct in some conditions, and not useful in others. Similarly, it remains to be seen what lidocaine's effect is when combined with other analgesics such as opioids and non-steroidal anti-inflammatory agents. This series does suggest that some patients with acutely painful conditions may benefit from intravenous lidocaine.

Limitations of this case series includes reliance on the accuracy and completeness of the written record. In a busy urban emergency department, physician and nursing charting may not accurately reflect the clinical reality. For instance, only 7 of the 17 patients had a pain score recorded both before and after the administration of lidocaine.

This type of review is also unable to control for bias and confounders, as there was no blinding of the patient, physician, or nurses. Cause and effect are also nearly impossible to establish, as the majority of patients received morphine prior to lidocaine, and improvement in their pain may have been due to the opioids, or at least a synergistic effect with the lidocaine.

This case series adds to the small but growing body of literature that supports lidocaine's judicious use as part of a multi-modal analgesia approach to acute pain in the emergency department. Future studies should continue to evaluate intravenous lidocaine's role in treating a variety of painful conditions in the emergency setting. Acute fracture pain is one area in which lidocaine may be of benefit, as there is an acute injury that may be susceptible to its sodium blockade of injured tissue.

\section{CONFLICT OF INTEREST}

No potential conflict of interest relevant to this article was reported.

\section{REFERENCES}

1. Guidelines 2000 for Cardiopulmonary Resuscitation and Emergency Cardiovascular Care. Part 6: advanced cardiovascular life support: section 5: pharmacology I: agents for arrhythmias. The American Heart Association in collaboration with the International Liaison Committee on Resuscitation. Circulation 2000;102(8 Suppl):1112-28.

2. Salhi $B$, Stettner E. In defense of the use of lidocaine in rapid sequence intubation. Ann Emerg Med 2007;49:84-6.

3. Sharma S, Rajagopal MR, Palat G, Singh C, Haji AG, Jain D. A phase II pilot study to evaluate use of intravenous lidocaine for opioid-refractory pain in cancer patients. J Pain Symptom Manage 2009;37:85-93.

4. De Oliveira GS Jr, Fitzgerald P, Streicher LF, Marcus RJ, McCarthy RJ. Systemic lidocaine to improve postoperative quality of recovery after ambulatory laparoscopic surgery. Anesth Analg 2012;115:262-7.

5. Thomas J, Kronenberg R, Cox MC, Naco GC, Wallace $M$, von Gunten CF. Intravenous lidocaine relieves severe pain: results of an inpatient hospice chart review. J Palliat Med 2004;7: 660-7. 
6. Soleimanpour $H$, Hassanzadeh $K$, Mohammadi DA, Vaezi $H$, Esfanjani RM. Parenteral lidocaine for treatment of intractable renal colic: a case series. J Med Case Rep 2011;5:256.

7. Soleimanpour H, Hassanzadeh K, Vaezi H, Golzari SE, Esfanjani RM, Soleimanpour M. Effectiveness of intravenous lidocaine versus intravenous morphine for patients with renal colic in the emergency department. BMC Urol 2012;12:13.

8. Vahidi E, Shakoor D, Aghaie Meybodi M, Saeedi M. Comparison of intravenous lidocaine versus morphine in alleviating pain in patients with critical limb ischaemia. Emerg Med J 2015;32:516-9.

9. Roden DM. Antiarrhythmic drugs. In: Limbird LE, Gilman AG, editors. Goodman \& Gilman's the pharmacological basis of therapeutics. 10th ed. New York: McGraw-Hill; 2001. p. 961-2.

10. McCleane G. Intravenous lidocaine: an outdated or underuti- lized treatment for pain? J Palliat Med 2007;10:798-805.

11. Devor M, Wall PD, Catalan N. Systemic lidocaine silences ectopic neuroma and DRG discharge without blocking nerve conduction. Pain 1992;48:261-8.

12. Rosen N, Marmura M, Abbas M, Silberstein S. Intravenous lidocaine in the treatment of refractory headache: a retrospective case series. Headache 2009;49:286-91.

13. Wasiak J, Spinks A, Costello V, et al. Adjuvant use of intravenous lidocaine for procedural burn pain relief: a randomized double-blind, placebo-controlled, cross-over trial. Burns 2011; 37:951-7.

14. Tanen DA, Shimada M, Danish DC, Dos Santos F, Makela M, Riffenburgh $\mathrm{RH}$. Intravenous lidocaine for the emergency department treatment of acute radicular low back pain, a randomized controlled trial. J Emerg Med 2014;47:119-24. 